\title{
Optical Sum Frequency Generation Spectroscopy of Cracked Non-Glutinous Rice (Oryza sativa L.) Kernels
}

\author{
Wataru Kouyama ${ }^{1}$, Takahiro Nishida1, Khuat Thi Thu Hien ${ }^{1}$, Goro Mizutani ${ }^{*}$, \\ Hiroshi Hasegawa ${ }^{2}$, Hiroaki Miyamura ${ }^{3}$ \\ ${ }^{1}$ School of Materials Science, Japan Advanced Institute of Science and Technology, Nomi, Japan \\ ${ }^{2}$ School of Environmental Science, University of Shiga Prefecture, Hikone, Japan \\ ${ }^{3}$ Shiga Prefecture Agricultural Technology Promotion Center, Omihachiman, Japan \\ Email: *mizutani@jaist.ac.jp
}

Received 20 September 2015; accepted 3 January 2016; published 6 January 2016

Copyright (C) 2016 by authors and Scientific Research Publishing Inc.

This work is licensed under the Creative Commons Attribution International License (CC BY).

http://creativecommons.org/licenses/by/4.0/

cc) (7)

\begin{abstract}
In order to study the correlation between the cracking of rice (Oryza sativa L.) kernels and the molecular structure of the amylopectin in them, we attempted optical sum frequency generation (SFG) spectroscopy in the C-H stretching vibration region for normal and cracked japonica nonglutinous rice kernels. The samples were Koshihikari and Nipponbare. In Nipponbare, the width of the SFG spectrum peak at $2915 \mathrm{~cm}^{-1}$ of the cracked rice kernels was broader than that of the normal ones, while for Koshihikari there was no clear difference. The width of the $2915 \mathrm{~cm}^{-1}$ peak is suggested to originate from the variety of the higher-order structure of the saccharide chains in amylopectin. Although this is a tentative result, this method is shown to have a potential of serving for preventing the cracking of the rice kernels.
\end{abstract}

\section{Keywords}

Rice Kernels, Cracking in Hot Weather, Optical Sum Frequency Generation (SFG), Amylopectin, Non-Glutinous Rice

\section{Introduction}

High temperature in summer causes rice (Oryza sativa L.) kernels to crack and eventually break into pieces in-

"Corresponding author.

How to cite this paper: Kouyama, W., Nishida, T., Hien, K.T.T., Mizutani, G., Hasegawa, H. and Miyamura, H. (2016) Optical Sum Frequency Generation Spectroscopy of Cracked Non-Glutinous Rice (Oryza sativa L.) Kernels. Journal of Biomaterials and Nanobiotechnology, 7, 13-18. http://dx.doi.org/10.4236/ibnb.2016.71002 
side the chaffs [1]. Such bad rice gives a big economic damage to farmers and the investigation and removal of such problems are very important. The occurrence of the cracked rice kernels depends on the weather condition within 10 days after the panicle emergence, or at the early stage of grain filling [1]. Therefore, the cause of the cracked rice occurrence is suggested to be irregular development of starch in the rice kernels.

Starch is formed when $\alpha$-D-glucose is polymerized by enzymatic reaction. In general, the starch is composed of two components of homopolymers: amylose, a linear polymer of 1000 linked glucose molecules, and amylopectin, a high branching polymer of 10,000 - 100,000 linked glucose molecules [2] [3]. The amylopectin molecule has complicated branch linkages and its molecular weight is much larger than that of amylose. Thus, the molecule allows for a lot of functional properties according to the variety of its structure [4].

Sum frequency generation (SFG) is a second order nonlinear optical effect excited by two laser beams with visible and infrared wavelength. This effect occurs in a non-centrosymmetric structured medium by coherent optical excitation at two different frequencies. Biomaterials show SFG response because they have chiral or non-centrosymmetric structures. Hence SFG was used to analyze collagen in a fish scale [5] and keratin proteins in human hair [6], for example. C-H vibrational peak near $2900 \mathrm{~cm}^{-1}$ in the SFG spectra from amylopectin has a special line shape so that the SFG should be useful in analyzing starch in plants [7] [8]. The output light frequency is a sum of those of the two incident light fields. In this study the wavelengths of the two incident light fields were $532 \mathrm{~nm}$ and around $3000 \mathrm{~nm}$. The sum frequency generation spectroscopy is a vibrational spectroscopy such as infrared spectroscopy because it uses infrared light fields in resonance with molecular vibrations in the sample material.

We used cracked non-glutinous rice kernels harvested in 2011 in Shiga Prefecture, Japan, as samples in this study. We compared the SFG spectra of cracked rice kernels with those of normal ones and inferred that there may be a correlation between the cracking of rice kernels and the molecular structure of the amylopectin in them. This is a first trial step for clarifying the physical or chemical origin of the cracking of rice kernels by nonlinear vibrational spectroscopy in order to judge if the method has a potential for such purpose.

\section{Materials and Methods}

We used two types of non-glutinous japonica rice cultivar, Koshihikari and Nipponbare, which were cultivated at the Shiga Prefecture Agricultural Technology Promotion Center $\left(35^{\circ} 10^{\prime} 28^{\prime \prime} \mathrm{N}, 136^{\circ} 07^{\prime} 52^{\prime \prime E}\right)$, Shiga, Japan. Koshihikari is known to be susceptible to heat-induced grain cracking [9] [10]. Nipponbare is more resistant to heat-induced grain cracking than Koshihikari [9] [10]. These samples were harvested on September 8 (Koshihikari) and 28 (Nipponbare), 2011. That was well after the grain filling was completed. In the summer of 2011, the temperature was higher than that in the average year by $2^{\circ} \mathrm{C}$ [11]. At 10 days after the start of the grain filling, the cracking occurred for $61.3 \%$ and $10.8 \%$ of the rice kernels for Koshihikari and Nippobare, respectively. At the time of the complete grain filling, the cracking had occurred for nearly $80 \%$ of the rice kernels of the both two species. The sample kernels were taken from the rice plants cultivated in the same field.

Normal and cracked rice kernels were separated by an optical microscope among the kernels of both Koshihikari and Nipponbare. Photographic images of normal and cracked rice kernels of Koshihikari and Nipponbare are shown in Figure 1(a) and Figure 1(b), respectively. The crack lines are seen to run perpendicular to the long axes of the ellipsoidal outer shapes of the kernels and are indicated by yellow arrows. The samples were cut along the long axis of the kernels just as in Ref. [8] using a thin blade cutter and the SFG intensity from their crush cell layers between the endosperm and embryo on their cross-sections were measured. The crush cell layers were the best sampling position of SFG because it gives the strongest signal in the whole cross sectional area of the rice kernel [8]. The assumption here was that there should be an average change in the molecular structure in each kernel grown at high temperature. The cracking might have occurred due to the mechanical stress generated by this molecular change. In order to get one SFG spectrum of cracked rice kernels of Koshihikari, we selected two cracked kernels and measured SFG spectra at two different positions on the crush cell layers. Thus each SFG spectrum in this paper is an average of four spectra from two samples. We obtained spectra of normal rice kernels of Koshihikari, and normal and cracked rice kernels of Nipponbare in the same way. Here we note that the "normal" rice kernels were grown in the same field as the cracked rice kernels. Measurement of the spectrum was performed by changing the wavenumber of the infrared light from $2750 \mathrm{~cm}^{-1}$ to $3150 \mathrm{~cm}^{-1}$. The total accumulation time at each wavenumber in each spectrum was $\sim 90$ sec. A polycrystalline ZnS pellet was used as the reference sample. 


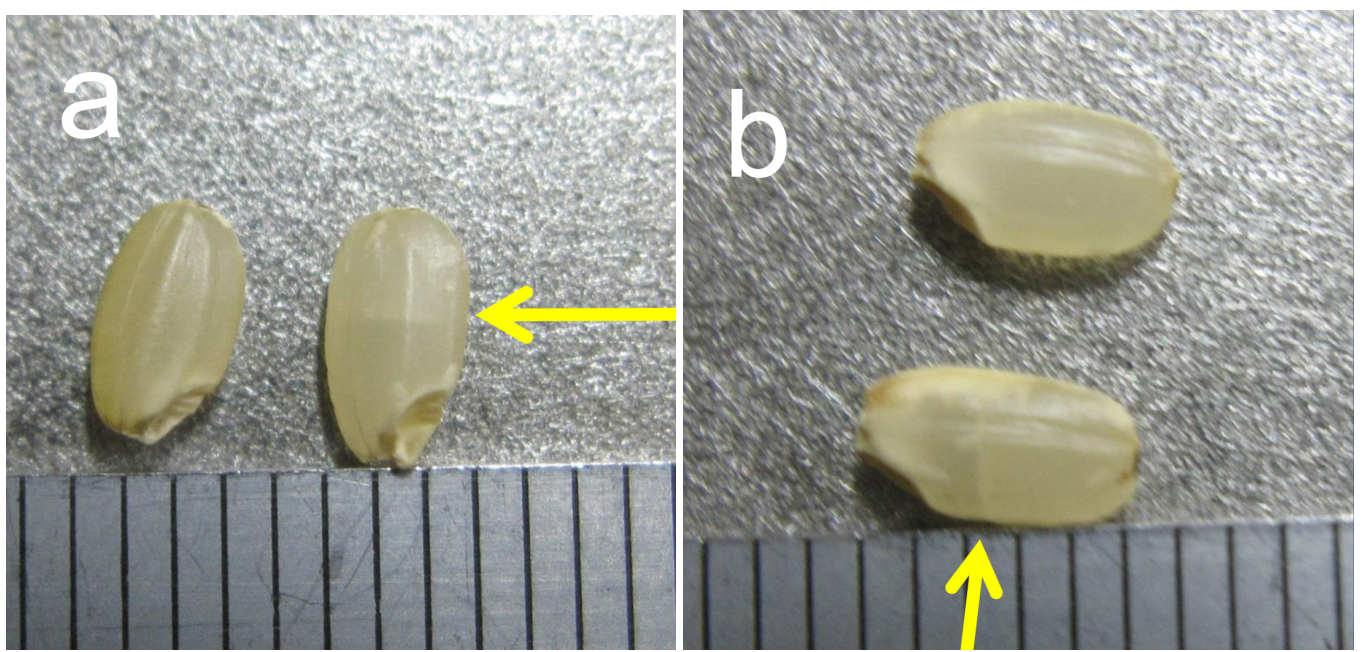

Figure 1. Photographs of normal and cracked rice kernels of (a) Koshihikari and (b) Nipponbare harvested on September 8 and 28 in year 2011 in Shiga prefecture Japan, respectively, taken by a normal camera. The cracks are indicated by the yellow arrows. The scale spacing is $1 \mathrm{~mm}$.

Figure 2 shows the optical setup of sum frequency generation confocal microscopy and spectroscopy system. One of the excitation light sources was a pico-second Nd:YAG laser with the repetition frequency of $10 \mathrm{~Hz}$ and the light pulses of wavelength $1064 \mathrm{~nm}$ were converted into visible light pulses of $532.1 \mathrm{~nm}$ wavelength by the harmonic unit. Infrared light pulses of $3 \mu \mathrm{m}$ wavelength was generated in the optical parametric generator/ difference frequency generator (OPG/DFG) system using the $1064 \mathrm{~nm}$ and $532 \mathrm{~nm}$ light. The bandwidth of this IR light is $6 \mathrm{~cm}^{-1}$ and this determines the spectral resolution of the SFG spectroscopy. This Infrared (IR) light was reflected by gold mirrors and focused onto the sample by a $\mathrm{CaF}_{2}$ lens of focal length of $200 \mathrm{~mm}$. The spot size of this IR light on the sample was from 100 to $200 \mu \mathrm{m}$. The visible light of photon energy $532 \mathrm{~nm}$ passed through the delay line and the neutral density filters, and was focused vertically on the sample by an objective lens of magnification $\times 20$ and the numerical aperture of 0.45 . The pulse energies were below $1 \mu \mathrm{J}$ for the visible pulses and $50 \mu \mathrm{J}$ for the IR light pulses. The SFG light pulses from the sample were collected by the objective lens, reflected by a series of mirrors and a dichroic mirror, passed through band pass filters, and were focused on a pinhole of $2 \mathrm{~mm}$ diameter and were detected by a photomultiplier (Hamamatsu R585). The center wavelength of the band pass filter was $460 \mathrm{~nm}$ with the half bandwidth (full width at half maximum of the transmission) of $10 \mathrm{~nm}$. The center wavelength $460 \mathrm{~nm}$ of the filter corresponds to that of the SFG light from the C-H stretching mode.

\section{Results and Discussion}

Figure 3(a) and Figure 3(b) are the SFG spectra of normal and cracked rice kernels of Koshihikari, respectively. Figure 4(a) and Figure 4(b) are those of Nipponbare. The SFG intensity was normalized to that from a reference $\mathrm{ZnS}$ polycrystalline pellet. In Figure 3 and Figure 4 the experimental data were fitted to a theoretical calculation equation with two resonances as [12],

$$
\left|\chi^{S F G}\right|^{2}=\left|\chi^{N R}+\frac{A_{1} \exp \left(i \theta_{1}\right)}{\left(x-\omega_{1}-i \gamma_{1}\right)}+\frac{A_{2} \exp \left(i \theta_{2}\right)}{\left(x-\omega_{2}-i \gamma_{2}\right)}\right|^{2}
$$

by a least square fitting method with a mathematical software IGOR [13]. $\chi^{S F G}$ is the nonlinear susceptibility of SFG, and $\chi^{N R}$ is a background term of non-resonant nonlinear susceptibility. $A_{1}$ and $A_{2}$ are constants, $\theta_{1}$ and $\theta_{2}$ are a phase difference between $\chi^{N R}$ and $A_{1}$ and $A_{2}, \gamma_{1}$ and $\gamma_{2}$ are peak widths, and $\omega_{1}$ and $\omega_{2}$ are peak positions for resonances. In Table 1 we list the best fit parameters of $A, \theta, \gamma$, and $\omega$ for the SFG spectra in Figure 3 and Figure 4. Among the parameters in Table 1 obtained by the fitting, $\gamma$ 's are important in our study because they reflect the spread of the C-H vibration frequencies caused by the variety of the saccharide chains in the amylopectin structures. 
IR: $3000 \mathrm{~nm}$

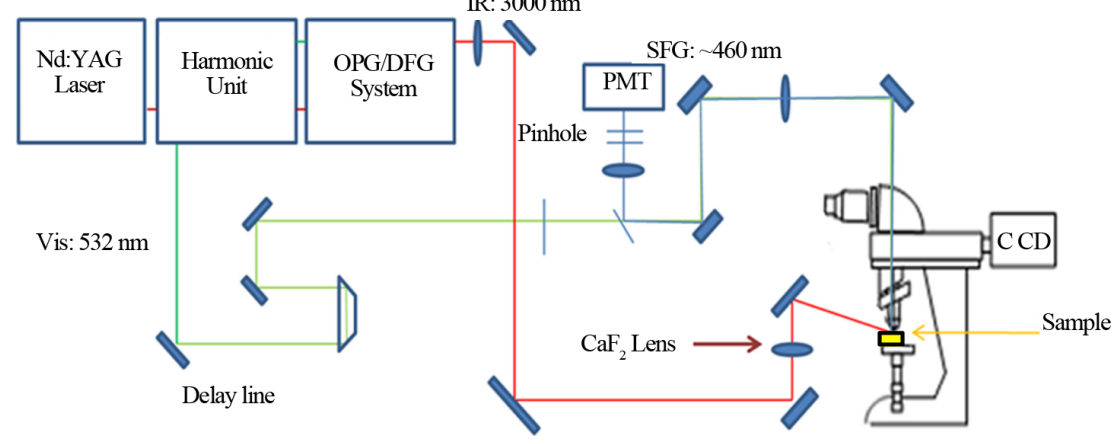

Figure 2. Experimental set up. OPG and DFG represent optical parametric generator and difference frequency generator, respectively. PMT represents photomultiplier tube. CCD represents charge-coupled device.

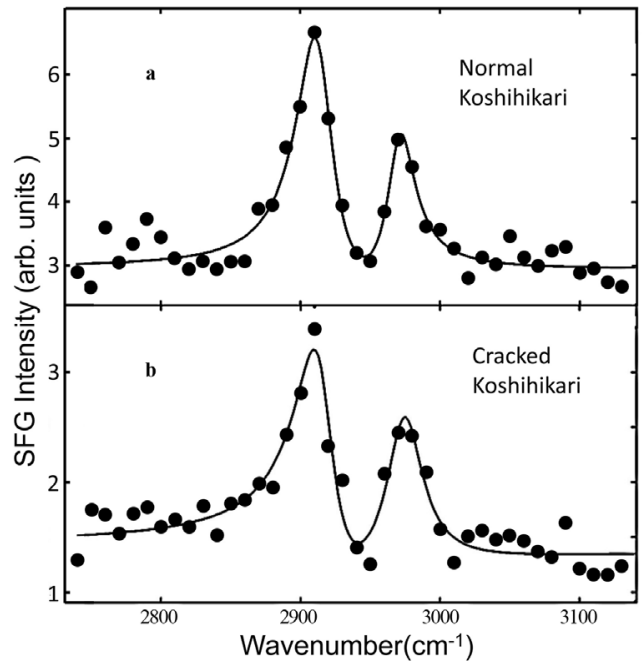

Figure 3. SFG spectra (a) of normal rice and (b) of cracked rice of Oryza sativa L.cv. Koshihikari.

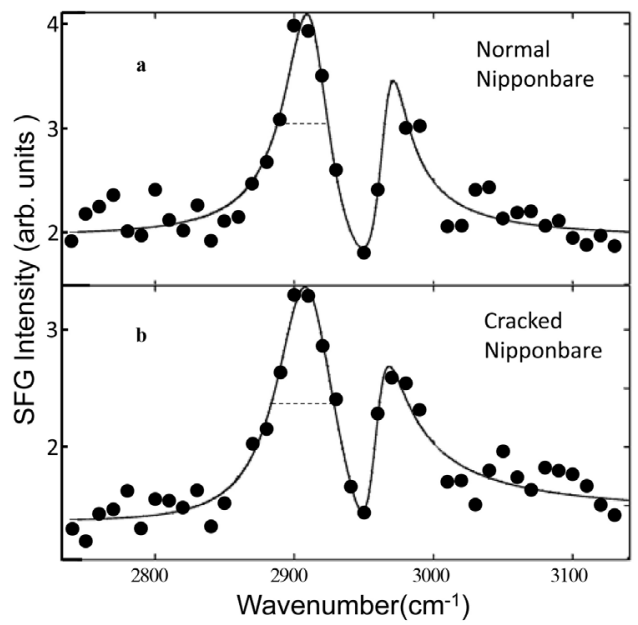

Figure 4. SFG spectra (a) of normal rice and (b) of cracked rice of Oryza sativa L.cv. Nipponbare. As a guide to the eye, dotted lines are drawn at the half heights of the 2915 $\mathrm{cm}^{-1}$ peaks with respect to the left tail ends of the spectra. The lengths of the dotted lines are $36.1 \mathrm{~cm}^{-1}$ for (a) and $45.1 \mathrm{~cm}^{-1}$ for (b). 
Table 1. Fitted parameters of resonance of $\mathrm{C}-\mathrm{H}$ stretching vibrations from the SFG spectra of normal and cracked rice kernels.

\begin{tabular}{|c|c|c|c|c|}
\hline & $\begin{array}{l}\text { Koshihikari } \\
\text { Normal rice }\end{array}$ & $\begin{array}{l}\text { Koshihikari } \\
\text { Cracked rice }\end{array}$ & $\begin{array}{l}\text { Nipponbare } \\
\text { Normal rice }\end{array}$ & $\begin{array}{l}\text { Nipponbare } \\
\text { Cracked rice }\end{array}$ \\
\hline Nonlinear susceptibility $\chi^{N R}$ & $1.2 \pm 0.01$ & $0.8 \pm 0.01$ & $0.9 \pm 0.01$ & $0.8 \pm 0.01$ \\
\hline Constant parameter $A_{1}$ & $19.3 \pm 2.6$ & $15.9 \pm 3.1$ & $19.3 \pm 3.1$ & $25.1 \pm 3.5$ \\
\hline Peak position $\omega_{1}\left(\mathrm{~cm}^{-1}\right)$ & $2913.7 \pm 2.0$ & $2916.0 \pm 2.5$ & $2913.9 \pm 2.7$ & $2909.5 \pm 3.6$ \\
\hline Peak width $\gamma_{1}\left(\mathrm{~cm}^{-1}\right)$ & $16.2 \pm 2.1$ & $16.9 \pm 2.9$ & $20.7 \pm 3.0$ & $26.5 \pm 3.3$ \\
\hline Phase difference $\theta_{1}\left(^{\circ}\right)$ & $233.5 \pm 10.7$ & $203.2 \pm 14.5$ & $235.0 \pm 12.3$ & $266.9 \pm 13.2$ \\
\hline Constant parameter $A_{2}$ & $36.8 \pm 8.0$ & $34.1 \pm 7.8$ & $29.6 \pm 6.5$ & $19.7 \pm 7.5$ \\
\hline Peak position $\omega_{2}\left(\mathrm{~cm}^{-1}\right)$ & $2970.1 \pm 3.1$ & $2974.5 \pm 3.7$ & $2966.5 \pm 3.2$ & $2958.7 \pm 2.9$ \\
\hline Peak width $\gamma_{2}\left(\mathrm{~cm}^{-1}\right)$ & $12.1 \pm 2.9$ & $16.0 \pm 4.1$ & $12.4 \pm 2.7$ & $12.2 \pm 3.6$ \\
\hline Peak Phase $\theta_{2}\left({ }^{\circ}\right)$ & $75.4 \pm 4.5$ & $81.5 \pm 6.3$ & $64.3 \pm 6.1$ & $48.0 \pm 15.8$ \\
\hline Phase difference $\theta_{1}-\theta_{2}\left({ }^{\circ}\right)$ & $158.1 \pm 14.1$ & $118.8 \pm 22.4$ & $163.7 \pm 11.9$ & $202.6 \pm 13.4$ \\
\hline
\end{tabular}

All the four SFG spectra have two peaks at $\sim 2915 \mathrm{~cm}^{-1}$ and $\sim 2970 \mathrm{~cm}^{-1}$ and one dip at $\sim 2950 \mathrm{~cm}^{-1}$. These two peaks and especially the dip between them are characteristic of the SFG spectrum of amylopectin [7], so the observed SFG signal is mainly assigned to amylopectin. The peak at $\sim 2915 \mathrm{~cm}^{-1}$ is assigned to a C-H vibrational mode and the one at $\sim 2970 \mathrm{~cm}^{-1}$ is assigned to a C- $\mathrm{H}_{2}$ vibrational mode [7] [14]. There was a difference in the width of SFG spectrum between Koshihikari and Nipponbare. According to Table 1, the fitted width of the C-H resonance peak at $\sim 2915 \mathrm{~cm}^{-1}$ is $\gamma_{1}=16.2 \pm 2.1 \mathrm{~cm}^{-1}$ for the normal Koshikari rice kernels and $\gamma_{1}=16.9 \pm 2.9$ $\mathrm{cm}^{-1}$ for cracked Koshihikari rice kernels. They are similar to each other within the statistical error. As for Nipponbare, the fitted width of the C-H resonance peak at $2915 \mathrm{~cm}^{-1}$ is $\gamma_{1}=20.7 \pm 3.0 \mathrm{~cm}^{-1}$ for normal rice and $\gamma_{1}$ $=26.5 \pm 3.3 \mathrm{~cm}^{-1}$ for cracked rice. The difference is not statistically distinct, but the latter tends to be larger than the former. As a guide to the eye in Figure 4, dotted lines are drawn at the half heights of the $2915 \mathrm{~cm}^{-1}$ peaks with respect to the left tail ends of the spectra. The lengths of the dotted lines are $36.1 \mathrm{~cm}^{-1}$ for (a) and $45.1 \mathrm{~cm}^{-1}$ for (b). The fitted peak width of the C- $\mathrm{H}_{2}$ resonance at $\sim 2970 \mathrm{~cm}^{-1}$ seems to be similar to each other within the statistical error for all the four spectra.

Summarizing all the above results, the width of the SFG spectrum peak at $2915 \mathrm{~cm}^{-1}$ of the cracked rice kernels of Nipponbare was broader than that of the normal ones, while for Koshihikari there was no clear difference. There was no difference in the widths of the peaks at $2970 \mathrm{~cm}^{-1}$.

The SFG spectra in the C-H stretching region near $3000 \mathrm{~cm}^{-1}$ of glucose and polysaccharides give different peak structures according to Refs. [7] and [15]. For example, the spectrum peak width of the C-H stretching mode at $2920 \mathrm{~cm}^{-1}$ of $\beta$-cyclodextrin is wider than that of glucose because dextrin molecules have more complex molecular structures [7]. Thus, it is tentatively inferred that the broadening of the peak at $2920 \mathrm{~cm}^{-1}$ occurs due to the modulation of the orientation order of the saccharide chains in the amylopectin of the cracked rice of Nipponbare. The validity of this picture should be checked in the future. It is widely said that higher environment temperature tends to increase the amount of longer amylopectin chains and decrease that of shorter chains [16]. The reason why the difference was not clear for Koshihikari is unknown but it at least may be that the modulation due to the temperature was not so big as the variety of the structure of the normal rice.

As a future prospective, it is indispensable to monitor various parts in the cracked rice kernels by SFG spectrscopy and microscopy and find out the variation of the property of amylopectin in the rice kernels. It may be possible to predict whether the rice kernels of Nipponbare will be cracked or not, by monitoring the SFG spectra before the grain filling stage when hot summer is expected.

\section{Conclusion}

We have compared the SFG spectra of the normal and cracked rice kernels of Koshihikari and Nipponbare. As a result, we have found an increase in the peak width of the C-H vibration peak at $2915 \mathrm{~cm}^{-1}$ in cracked rice kernels of Nipponbare. The width is suggested to originate from the variety of the higher-order structure of the 
saccharide chains in amylopectin. It will be necessary to measure more samples of other cultivars for the establishment of the results. However, the approach in this study may contribute to the elucidation of the origins of the rice cracking. This result will become the first step to the comprehension of the amylopectin's structure in unusual rice kernels.

\section{References}

[1] Nagata, K., Takita, T., Yoshinaga, S., Terashima, K. and Fukuda, A. (2004) Effect of Air Temperature during the Early Grain-Filling Stage on Grain Fissuring in Rice. Japanese Journal of Crop Science, 73, 336-342. http://dx.doi.org/10.1626/jcs.73.336

[2] Heldt, H.W. (1999) Pflanzenbiochemie. 2nd Edition, Chapter 9, Spektrum Akademischer Verlag GmbH, Heidelberg Berlin.

[3] Tester, R.F., Karkalas, J. and Qi, X. (2003) Starch-Composition, Fine Structure and Architecture. Journal of Cereal Science, 39, 151-165. http://dx.doi.org/10.1016/j.jcs.2003.12.001

[4] Nakamura, Y., Francisco, P.B., Hosaka, Y., Sato, A., Sawada, T., Kubo, A. and Fujita, N. (2005) Essential Amino Acids of Starch Synthase IIa Differentiate Amylopectin Structure and Starch Quality between Japonica and Indica Rice Varieties. Plant Molecular Biology, 58, 213-227. http://dx.doi.org/10.1007/s11103-005-6507-2

[5] Kouyama, W., Ogawa, A., Li, H., Miyauchi, Y., Mizutani, G. and Sano, H. (2014) Sum Frequency Generation Confocal Microscopy Observation of a Fish Scale. e-Journal of Surface Science and Nanotechnology, 12, 259-262. https://www.jstage.jst.go.jp/article/ejssnt/12/0/12_259/_pdf http://dx.doi.org/10.1380/ejssnt.2014.259

[6] Sakai, M., Kikuchi, K. and Fujii, M. (2013) Quaternary and Secondary Structural Imaging of a Human Hair by VSFGDetected IR Super-Resolution Microscope. Chemical Physics, 419, 261-265.

http://dx.doi.org/10.1016/j.chemphys.2013.02.016

[7] Miyauchi, Y., Sano, H. and Mizutani, G. (2006) Selective Observation of Starch in a Water Plant Using Optical Sum Frequency Microscopy. Journal of the Optical Society of America A, 23, 1687-1690. http://dx.doi.org/10.1364/JOSAA.23.001687

[8] Li, H., Miyauchi, Y., Tuan, N.A., Mizutani, G. and Koyano, M. (2012) Optical Sum Frequency Generation Image of Rice Grains. Journal of Biomaterials and Nanobiotechnology, 3, 286-291. http://dx.doi.org/10.4236/jbnb.2012.322035

[9] Shiga Prefecture Agricultural Technology Promotion Center. http://www.pref.shiga.lg.jp/g/nogyo/files/ondankataisaku.pdf

[10] Nagata, K., Sasaki, R. and Ohdaira, Y. (2013) Cultivar Difference in the Grain Cracking of Rice under the High Air Temperature Condtins during Grain Filling. Japanese Journal of Crop Science, 73, 42-48. http://dx.doi.org/10.1626/jcs.82.42

[11] Japan Meteorological Agency Hikone Local Meteorological Office (2011) http://www.data.jma.go.jp/obd/stats/etrn/view/monthly_s1.php?prec_no=60\&block_no=47761\&year=2011\&month=\& $\underline{\text { day }=\& \text { view }}=$ http://www.data.jma.go.jp/obd/stats/etrn/view/nml_sfc_ym.php?prec_no=60\&block_no=47761\&year=\&month=\&day $=\&$ view $=$

[12] Lu, G.Q., Lagutchev, A., Dlott, D.D. and Wieckowski, A. (2005) Quantitative Vibrational Sum-Frequency Generation Spectroscopy of Thin Layer Electrochemistry: CO on a Pt Electrode. Surface Science, 585, 3-16. http://dx.doi.org/10.1016/j.susc.2005.02.041

[13] http://www.wavemetrics.com/index.html

[14] Kong, L., Lee, C., Kim, S.H. and Ziegler, G.R. (2014) Characterization of Starch Polymorphic Structures Using Vibrational Sum Frequency Generation Spectroscopy. Journal of Physical Chemistry B, 118, 1775-1783. http://dx.doi.org/10.1021/jp411130n

[15] Hieu, H.C., Tuan, N.A., Li, H., Miyauchi, Y. and Mizutani, G. (2011) Sum Frequency Generation Microscopy Study of Cellulose Fibers. Applied Spectroscopy, 65, 1254-1259. http://dx.doi.org/10.1366/11-06388

[16] Asaoka, M. (1984) Effect of Environmental Temperature during Development of Rice Plants on Some Properties of Endosperm Starch. Starch/Staerke, 36, 189-193. http://dx.doi.org/10.1002/star.19840360602 